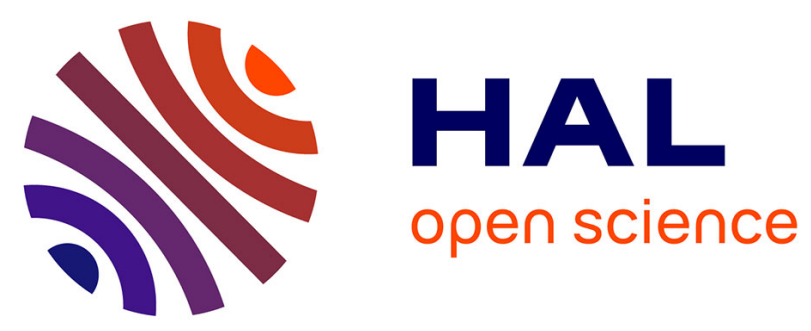

\title{
ProMeSCT: A Proximal Metric Algorithm for Spectral CT
}

\author{
Souhil Tairi, Sandrine Anthoine, Yannick Boursier, Mathieu Dupont, \\ Christian Morel
}

\section{- To cite this version:}

Souhil Tairi, Sandrine Anthoine, Yannick Boursier, Mathieu Dupont, Christian Morel. ProMeSCT: A Proximal Metric Algorithm for Spectral CT. IEEE Trans.Rad.Plasma Med.Sci., 2021, 5 (4), pp.548558. 10.1109/trpms.2020.3036028 . hal-03327374

\section{HAL Id: hal-03327374 https://hal.science/hal-03327374}

Submitted on 25 Oct 2021

HAL is a multi-disciplinary open access archive for the deposit and dissemination of scientific research documents, whether they are published or not. The documents may come from teaching and research institutions in France or abroad, or from public or private research centers.
L'archive ouverte pluridisciplinaire HAL, est destinée au dépôt et à la diffusion de documents scientifiques de niveau recherche, publiés ou non, émanant des établissements d'enseignement et de recherche français ou étrangers, des laboratoires publics ou privés. 


\title{
ProMeSCT: a Proximal Metric Algorithm for Spectral CT
}

\author{
Souhil Tairi, Sandrine Anthoine, Yannick Boursier, Mathieu Dupont and Christian Morel
}

\begin{abstract}
The acquisition of a set of spectral Photon-Counting Computed Tomography (spectral PC-CT) measurements aims at uncovering both the spatial and energetic characteristics of the imaged body, which widens the potential of tomography compared to classical Computed Tomography (CT). In the preclinical context, the use of polychromatic beams induces spectral mixing and, as a consequence, the reconstruction procedure requires specific algorithmic tools more complex than the standard ones used in CT. In this paper, we propose a one-step inversion method to simultaneously separate and reconstruct the physical materials of an object observed in the context of spectral PC-CT. To do so, we carefully consider the underlying polychromatic model of the X-ray beam and combine it with $a$ priori on the materials of the object to reconstruct. The simultaneous separation and reconstruction of materials is done by minimizing the resulting non-convex ill-posed inverse problem. The dimensionality of the data and object materials worsens the computational complexity of the problem. We propose an efficient optimization algorithm based on a proximal forward-backward algorithm that is accelerated by a metric, which is specifically designed for spectral PC-CT. The efficiency of our method called ProMeSCT is demonstrated on results obtained on 3D synthetic data with a simple regularization that encompasses the positivity of the quantities of interest.
\end{abstract}

Index Terms-Photon-Counting CT, spectral CT, one-step inversion, proximal algorithm.

\section{INTRODUCTION}

X-ray Computed Tomography (CT) is an imaging modality that is widely used today in the medical context. A set of measurements called "projections" are acquired by rotating a couple X-ray source / detector array around the patient. This set of measurements is subsequently fed to a reconstruction process that allows to uncover a spatial map of the mass attenuation coefficient in the patient. This map reveals the internal structure of the patient and depending on the exam can be used to uncover e.g. bone trauma, calcification, tumors or hemorrhage.

Spectral Photon-Counting Computed Tomography (spectral PC-CT) is a new imaging modality that enhances the classical tomographic measurements (which give only spatial information) with energy-dependent (i.e. spectral) attenuation properties. The advantage is that this spectral information can be used to discriminate different physical phenomena such as Compton or photo-electric effect, or different materials in the patient such as soft-tissues, bones or contrast agents. This technology opens the way for using X-ray tomography as

S. Tairi, Y. Boursier, M. Dupont and C. Morel are with Aix Marseille Univ, CNRS/IN2P3, CPPM, Marseille, France.

S. Tairi and S. Anthoine are with Aix Marseille Univ, CNRS, Centrale Marseille, I2M, Marseille, France. a functional imaging tool, which can naturally improve for example the study of biomedical or clinical data.

The principles of spectral tomography have been demonstrated by Alvarez and Macovsky [1] in the seventies but the technology to make the acquisitions has been developed more recently. First, Dual Energy CT scanners (DECT) were deployed, they enable to acquire two sets of measurements using two different spectral bands [2] and offer the possibility to estimate two different materials without segmentation [3], [4]. In the last decade the advent of detectors working in PhotonCounting (PC) mode [5], [6], [7] has once more boosted the field, making it possible to acquire more than two sets of measurements with different spectral information by tuning the detectors to count photons in different energy bands. Current PC detectors may have one or several energetic thresholds that are tuned to acquire the information. Exploiting these spectral measurements allows to estimate more than two materials at the same time. The core concept is to view the material attenuation coefficient of the object as a linear combination of basis functions corresponding to known materials. With this assumption, any change in spectral measurements can be represented as a combination of material concentrations or lengths that cause it. By applying an adapted algorithm, we get a material distribution in the reconstructed volume.

Once a set of spectral PC-CT measurements is acquired, the challenges in the reconstruction process are twofold. i) As in classical CT one needs to reconstruct spatial properties from tomographic projections. Depending on the acquisition set-up or if one simply wants to reduce the radiation dose, one may acquire low-dose projections thus having a poor signal-to-noise ratio (SNR) or a reduced set of projections which may lead to an incomplete set. Generally speaking the tomographic reconstruction problem is by itself a difficult one, but it benefits from a large and solid literature from direct inversions method (e.g. FDK algorithm [8]) to more sophisticated regularized iterative ones [9], [10]. ii) One needs to exploit the spectral diversity in the different projections to disentangle the different materials one seeks. The fact that the contribution of each material changes according to the spectral configuration of the measure is what makes it possible to disentangle them. However the contribution of the materials typically mix in a non-linear fashion in the spectral PC-CT measurements. This non-linearity is one of the main reasons why the separation problem is difficult.

Both problems, the tomographic reconstruction and the spectral separation, are separately non-trivial by essence: they raise questions pertaining to the class of ill-posed inverse problems. In the literature, one can roughly separate the proposed 
methods into those that treat the reconstruction and separation problem sequentially (and thus separately) and those that treat both problems jointly, which are also referred to as "one-step inversion" methods. One-step inversion methods involve treating all the data and producing all the maps together, leading to a large dimensional problem that is computationally difficult. Treating the problems sequentially lightens the computational burden by breaking the problem into smaller pieces. The price to pay for this is that errors in the first step of the estimation process are propagated to the next, which may result in a lower robustness of the results. It also enables to rely on wellestablished tomographic reconstruction methods to treat the spatial reconstruction problem.

Sequential methods have been proposed first. Among these, some first handle the reconstruction problem and then proceed to the separation in the object space. This is the case for example for the pioneering K-edge methods [11], [12], [13], [14]. They leverage on the spectral profile of high-Z elements such as contrast agents, which have a characteristic spectral discontinuity (K-edge), to approximate the spectral separation by simple addition/subtraction. Currently, a number of methods are dedicated to reconstructing an attenuation map for each energy band that is acquired while enhancing the methods described above by jointly reconstructing those maps [15], [16], [4], [17]. In all the former cases, the nonlinear spectral mixing is not taken into account: one rather uses an approximate linear model. In order to use a model that is closer to the physics of the acquisition, others considered the spectral separation first, estimating thus the contribution of each material in measurement space (sinograms), and then proceeding to the spatial reconstruction of each map [18], [19], [20].

One-step inversion methods perform the separation and reconstruction simultaneously ( [3], [21], [22], [23], [24], [25], [26], [27]). They generally do so by setting-up a single inverse problem that takes into account all measurements and outputs all the material maps. The estimation is obtained by solving a minimization problem, which is done in an iterative fashion. As in the former cases however, a number of such methods are based on simplifying the non-linear mixing model. The approximation of the non-linear mixing usually appears when the authors design separable quadratic surrogates (SQS) [21], [22], [23]. In [21], several successive quadratic approximations are used while in [22], the authors rather use a predefined model of finite sums of exponentials that is calibrated beforehand. In [23], [3], [24], one remains closer to the physics of the acquisition, thus treating the non-linear mixing model. This is also the point of view adopted in this work. Solving a one-step inversion problem is computationally demanding. To alleviate this problem, different strategies are used. In [21], [22] and [23] the authors resort to SQS, which are easy to minimize at each iteration. Each iteration has thus a low computational cost but the non-convexity of the problem sometimes prevents from guaranteeing the global convergence of the procedure [21], [22]. Most of these methods regularize the problem, using $a$ priori on the reconstructed maps. While the choice of smooth regularizers as in [3] and [23] allows one to use classical optimization based on gradients, the choice of non-smooth regularizers, as those used in [24] renders the optimization problem more difficult but allows to sharpen the contrast of the objects contours. A very interesting practical work comparing the computational efficiency of different one-step inversion methods on two-dimensional synthetic data can be found in [26].

In this work, we present a one-step inversion method which is based on the minimization of a functional that seeks a balance between a fit-to-data term and a regularizer. As in [3], the fit-to-data is a direct transcription of the physics of the acquisition. It integrates the non-linear mixing effect described above. The a priori or regularizer is non-smooth as in [24]. The global optimization problem is non-smooth, non-convex and involves large-dimensional objects. As in [24], our method is based on the proximal operator [28], which is a tool used instead of the gradient when dealing with nonsmooth functions. The algorithm proposed in [24] is based on re-writing the functional to be minimized as a difference of convex functions and belongs to the family of "primaldual" algorithms. The algorithm consists in two nested loops and the convergence is guaranteed. In practice, the authors perform only one inner iteration, thus losing the convergence guarantee, and this procedure remains rather slow. By contrast, our method is based on a primal scheme that leverages on the use of a metric (which is similar to a preconditioner) to accelerate the convergence and that is provably convergent. This scheme, called VMILAn [29] belongs to the family of variable-metric forward-backward algorithms [30]. We design a metric tailored to the spectral PC-CT problem and show the efficiency on simulated and real data of resulting method called ProMeSCT.

The paper is organized as follows. In Section II, we derive the discretized model that links the measurements to the imaged object. It is based on the Beer-Lambert equation and a decomposition of the object into its basis materials. Section III is dedicated to the method proposed to perform the one-step inversion. We detail the minimization problem and the algorithm, with an emphasis on the design of the metric tailored to the problem. The last section is dedicated to experiments. We assess the performances of the proposed method on controlled synthetic data.

\section{THE PROBLEM OF SEPARATION AND RECONSTRUCTION IN SPECTRAL PC-CT}

In this section we define a mathematical framework that describes the links between the quantities we want to estimate and a generic set of spectral PC-CT measurements. In the formal model we establish here, the Beer-Lambert law is the fundamental link between the object and the measurements (see Section II-A). We also rely on a decomposition of the object as the sum of its materials (see Section II-B) and discretize the model in Section II-C.

\section{A. The forward model}

Let us start with the model linking the measurements to the object. Let $E$ be the X-ray photon energy and $x$ the spatial coordinate within the object space, and let us assume that: 
- The X-ray source is modulated by the presence of a filter (e.g. a metal), the resulting spectral profile is $I_{0}(E)$;

- The illuminated object is characterized by its attenuation coefficient $\mu(x, E)$;

- The detector has the spectral efficiency $\eta(E)$ and counts photons between the thresholds $E_{\text {min }}$ and $E_{\text {max }}$;

- $\mathcal{L}$ is the line of sight between the source and the detector.

Then a PC-CT detector ideally measures (Beer-Lambert law):

$$
\int_{E_{\min }}^{E_{\max }} I_{0}(E) \eta(E) \mathrm{e}^{-\int_{\mathcal{L}} \mu(x, E) d x} d E .
$$

In spectral PC-CT, one acquires several sets of measurements - say $M$ - that differ by their spectral inputs. Each set consists of $P$ measurements corresponding to all the detector pixels and source/object positions. For each set $m=1 \cdots M$, one chooses a specific spectral configuration by setting: the source voltage and filter $\left(I_{0}^{m}\right)$, and the energy thresholds $\left(E_{\text {min }}^{m}\right.$, $E_{\text {max }}^{m}$ ). Defining $\mathcal{F}_{p, m}(E)=I_{0}^{m}(E) \eta^{p}(E) \chi_{\left[E_{\text {min }}^{m}, E_{\text {max }}^{m}\right]}(E)$ where $\chi$ stands for the indicator function, a full set of ideal spectral-PC-CT data is:

$y_{p, m}=\int_{\mathbb{R}} \mathcal{F}_{p, m}(E) \mathrm{e}^{-\int_{\mathcal{L}_{p}} \mu(x, E) d x} d E, \quad 1 \leq p \leq P, \quad 1 \leq m \leq M$.

\section{B. The spatio-spectral map model of the object}

Naturally, one cannot recover a full spatial and spectral content of the object (i.e. the function $\mu(x, E)$ for every $x$ and $E$ ) with only $M P$ measurements. One needs additional assumptions. Here we assume that the object is made of different materials (tissues, bones, etc.), say $K$ such materials and that for each of them, the attenuation coefficient is simply the product of its interaction cross-section $\sigma_{k}(E)$ by its concentration at location $x, a_{k}(x)$ :

$$
\mu(x, E)=\sum_{k=1}^{K} a_{k}(x) \sigma_{k}(E) .
$$

In this work, we assume that we know beforehand which $K$ materials (soft tissues, bones, contrast agents) constitute the object and that we have access to their interaction cross-section $\sigma_{k}(E)$. The goal is to recover the $K$ spatial maps $a_{k}(x)$ from the set of $M P$ measurements in (1), and the knowledge of the spectral inputs for each configuration.

\section{Discretization}

The object is divided into $L$ voxels and the spectral axis into $N$ energy bins. Each spatial map $a_{k}$ is thus discretized as a vector of length $L$. These vectors are stored column-wise into the matrix $A$ of size $L \times K$. The discretized interaction-cross sections $\sigma_{k}$ of length $N$ are stored row-wise into the matrix $\Sigma$ of size $K \times N$. Here, we assume that the detectors efficiencies are identical $\left(\mathcal{F}_{p, m}\right.$ does not depend on $\left.p\right)$, reducing the spectral inputs to a vector of length $N$ for each configuration $m$. They form the columns of the matrix $F$ of size $N \times M$.

The discretized tomographic projection operator is stored in the matrix $S$ of size $P \times L$, and the integral with respect to energy is discretized as a Riemanian sum. The $M P$ ideal measurements described in Eq. (1) are altered in practice by noise (instrumental or of other type). Denoting by $\mathcal{B}$ all the possible perturbations, the full forward model reads:

$$
Y=\mathcal{B}\left(\mathrm{e}^{-S A \Sigma} F\right)
$$

where $Y$ is a matrix of size $P \times M$ and $\mathrm{e}^{Z}$ stands for the matrix of the same size as $Z$ whose entries are the exponential of the entries of $Z$.

\section{PROMESCT, A FAST PROXIMAL ALGORITHM FOR SPECTRAL PC-CT}

\section{A. Inverse Problem}

Our goal in this paper is to recover the spatial maps of the $K$ materials stored in the matrix $A$ from the $M$ sets of measurements in $Y$, the spectral profiles of the materials stored in the matrix $\Sigma$, the spectral inputs stored in the matrix $F$ and a noise model $\mathcal{B}$, all of this taking into account the forward model in Eq. (3).

We tackle this inverse problem by minimizing a functional $J$ that seeks a balance between a discrepancy term $G(A)$ that measures how much the estimated maps $A$ explain the data $Y$ and a regularization term $R(A)$. We consider the discrepancy terms $G(A)$ of the form $G(A)=D\left(Y, e^{-S A \Sigma} F\right)$ with $D$ a function linked to the noise model $\mathcal{B}$. If $\mathcal{B}$ denotes a statistical noise model, $D$ corresponds to its negative log-likelihood. Here we will develop the cases of

- independent identically distributed Gaussian noise of unknown variance which leads to the mean squared error

$$
G_{G}(A)=\sum_{p, m}\left(Y-e^{-S A \Sigma} F\right)_{p, m}^{2},
$$

- Poisson noise on each measure $\left(e^{-S A \Sigma} F\right)_{p, m}$ which leads to the Kullback-Leibler divergence

$$
G_{P}(A)=\sum_{p, m}\left(e^{-S A \Sigma} F\right)_{p, m}-Y_{p, m} \log \left(e^{-S A \Sigma} F\right)_{p, m}
$$

Minimizing only the discrepancy term (which corresponds to finding the maximum-likelihood estimator in the above cases) usually leads to unstable solutions with respect to the perturbation of the measurements. This is well-known even when the forward model is linear and it is true also here where the forward model includes strong non-linearities. Adding a regularization term $R(A)$ is a classical solution to alleviate this problem. When it has good mathematical properties, $R$ reduces the set of minimizers of $J$ and forces robustness. The preferred solutions are those reproducing well the data and having a low value of $R$. By encoding in $R$ a priori we have on the spatial maps, we force the solutions to be both stable and realistic. Here we consider $R$ of the form:

$$
R(A)=R_{+}(A)+R^{\prime}(A)
$$

where the first term enforces the non-negativity of the concentration values in $A\left(R_{+}(A)=0\right.$ if $A_{l, k} \geq 0$ for all $(l, k)$ and $R_{+}(A)=+\infty$ otherwise) and $R^{\prime}$ forces spatial properties like smoothness or sparsity of each of the maps.

Let us note that the two discrepancy terms $G_{G}(A)$ and $G_{P}(A)$ are smooth (twice continuously differentiable) but not 
convex. This will be the case for most functions of the form $G(A)=D\left(Y, e^{-S A \Sigma} F\right)$. On the other hand, the examples of $R$ given above are convex but not smooth. More generally, the methodology we develop afterwards is tailored to handle a functional of the form $J(A)=G(A)+R(A)$ with $G$ a smooth (at least once continuously differentiable) function that is not necessarily convex and $R$ a convex but not necessarily differentiable function.

\section{B. A proximal metric algorithm: VMILAn}

The minimization of non-differentiable but convex functionals has received quite a lot of attention in the last two decades. Proximal algorithms in particular are a class of methods that have been developed successfully, with strong theoretical guarantees including convergence and optimal convergence rates along with various strategies to split and exploit the different parts of a convex functionals (see [31] for a survey). Lately, a subset of these proximal methods have been shown to preserve their mathematical properties when applied to nonconvex functionals. This is the case for example when the nonconvex part has the Kurdyka-Łojasiewicz (KŁ) property [32], [33], which essentially means that it can be re-parameterized to be steep around its minima. In this paper, we use one such proximal algorithm, VMILAn [29], to solve our problem.

1) Proximal algorithms on convex functionals: Let us first examine the case of convex functionals. A classical algorithm to minimize a convex functional $J=G+R$ with $G$ smooth and convex and $R$ non-smooth and convex is the ForwardBackward [34] algorithm that proceeds in two steps at each iteration:

- Forward step: gradient descent on $G$

$$
x^{i+1 / 2}=x^{i}-\gamma_{i} \nabla G\left(x^{i}\right)
$$

- Backward step: proximal descent on $R$

$$
x^{i+1}=\operatorname{prox}_{R}\left(x^{i+1 / 2}\right),
$$

where the proximal operator is defined by:

$$
\operatorname{prox}_{R}(x)=\operatorname{argmin}_{z} \frac{1}{2}\|z-x\|_{2}^{2}+R(z) .
$$

Note that the proximal operator is defined on convex functions only. The Forward-Backward scheme (FB) is quite easy to implement when the proximal operator is explicit, which is the case for a number of classical terms such as box constraints or the $l_{2}$, Thikonov and $l_{1}$ (or sparse) regularizations. For other terms such as the Total-Variation (TV) norm, efficient schemes have been derived. However the convergence rate is slow and has been sped up with two strategies: one is the multi-step strategy [35], [36] (also called Nesterov's momentum), and the other one is to introduce variable metrics [30], [29].

The multi-step strategy consists in choosing a point that will go a bit further away than the proximal point in the Backward step of the FB scheme using the previous iterates. Choosing carefully the amount of deviation, one can guarantee a fast convergence of the objective values $\left(\lim _{i} J\left(x^{i}\right)=\min J\right)$ [36] and sometimes the iterates $\left(\lim _{i} x^{i} \in \operatorname{argmin} J\right.$ where $\operatorname{argmin}$ denotes the set of global minimizers) [37] for convex functionals only, that is when $J, G$ and $R$ are convex. Several such schemes exist, the classical FISTA [36] is described in Algo. 1.

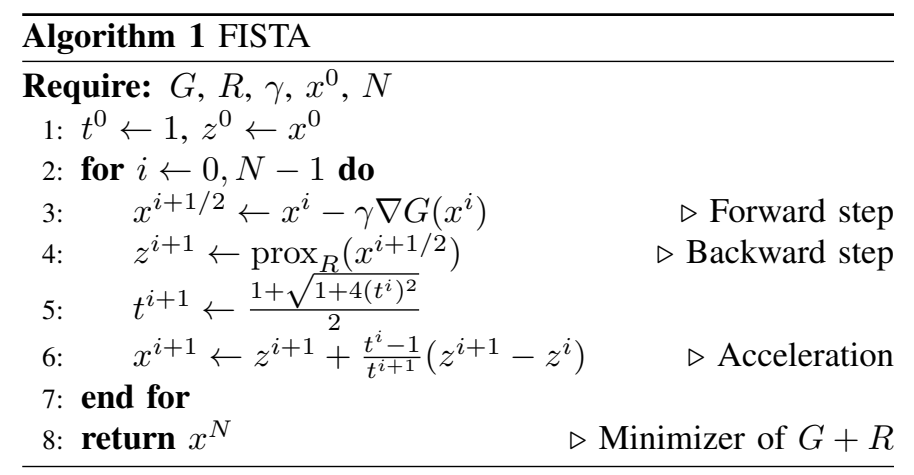

A variable metric proximal scheme preconditions the gradient descent on $G$ at each iteration by a definite-positive matrix $\Xi_{i}$ and accordingly modifies the proximal step:

- Forward step: $x^{i+1 / 2}=x^{i}-\gamma_{i} \Xi_{i}^{-1} \nabla G\left(x^{i}\right)$,

- Backward step: $x^{i+1}=\operatorname{prox}_{\Xi_{i}, R}\left(x^{i+1 / 2}\right)$,

where the metric proximal operator is defined by:

$$
\begin{aligned}
\operatorname{prox}_{\Xi, R}(x) & =\underset{z}{\operatorname{argmin}} \frac{1}{2}\|z-x\|_{\Xi}^{2}+R(z) \\
& =\underset{z}{\operatorname{argmin}} \frac{1}{2}\langle z-x, \Xi(z-x)\rangle+R(z) .
\end{aligned}
$$

In the convex case, that is when $J, G$ and $R$ are convex, the convergence of the variable metrics scheme holds both in terms of objective values $\left(\lim _{i} J\left(x^{i}\right)=\min J\right)$ and iterates $\left(\lim _{i} x^{i} \in \operatorname{argmin} J\right)$. Notice that the variable metrics scheme reduces to $\mathrm{FB}$ when $\Xi_{i}$ is the identity. Hence the acceleration that is obtained depends naturally on the metrics that are chosen.

2) Proximal algorithms on non-convex functionals: Let us now turn to the case of non-convex functionals as the one of interest here. Non-convex functional may have local and global minima. The proximal method described above are first order algorithms (i.e. they depend only on the first differential). One can generally not ensure that such an algorithm reaches a global minimizer. Nevertheless, one can ensure that the sequence of iterates converges to a critical point - which is a point that zeroes the first differential.

Proximal methods were originally designed for convex functionals. Seeing their success, scientists naturally tried them in practice on non-convex functionals obtaining sometimes results of good quality. This is what we show also here with the multi-step strategies (or Nesterov's momentum) in the experimental section. However, the convergence of multi-step strategies cannot be mathematically proved neither in terms of iterates nor in terms of objective values. One is thus not able to guarantee their good behavior: the sequence of iterates could diverge (coordinate blowing to infinity or cycling on a subspace), and the sequence of objective values is also not monotonic and could diverge, etc.

FB and variable metric schemes on the other hand are provably convergent on a class of non-convex functionals including that of interest here, which means that both the objective values and the iterates converge to a limit, and that the limit point of the iterates is a critical point. Moreover, the sequence of objective values is non-increasing, which maximizes the chances to reach a global minimizer. This is 
the reason why we advocate to use them here. More precisely, the convergence is guaranteed if $J$ is a non-convex functional that can be written as $J=G+R$ with $R$ convex (but non-smooth) and $G$ smooth (but non-convex) and having the Kurdyka-Łojasiewicz (KŁ) property [32], [33]. The KŁ property essentially means that one can control the function growth with its differential. The set of functions having this property is quite large, including for example the set of realanalytic functions. Notice that in the variable metrics scheme, the proximal operator is computed on the convex part of the functional $R$.

3) VMILAn in practice: The increase of speed introduced by the metric compared to FB has to be balanced with the increase of complexity of the proximal point computation. VMILAn provides an implementation of the variable metrics scheme when only an approximation of the solution of the proximal problem $x^{i+1}=\operatorname{prox}_{\Xi_{i}, R}\left(x^{i+1 / 2}\right)$ is available. The precision of the approximation is quantified and can be computed in practice. VMILAn is guaranteed to converge to a critical point of $J$ when this precision is reached and under technical conditions ${ }^{1}$ [29]. The complete VMILAn algorithm is described in Algo. 2. After the initialization of various quantities; Line 3 shows the choice of the gradient step at each iteration, bounded above and below by quantities defined above; Line 4 shows the choice of the metric (positivedefinite matrix) at each iteration, its spectral norm (maximal eigenvalue) must also be bounded by predefined quantities; Line 5 proceeds to the modified gradient step on $G$ and Line 6 to the proximal step, the Approx operator denoting the fact that only an approximation of the proximal point is required.

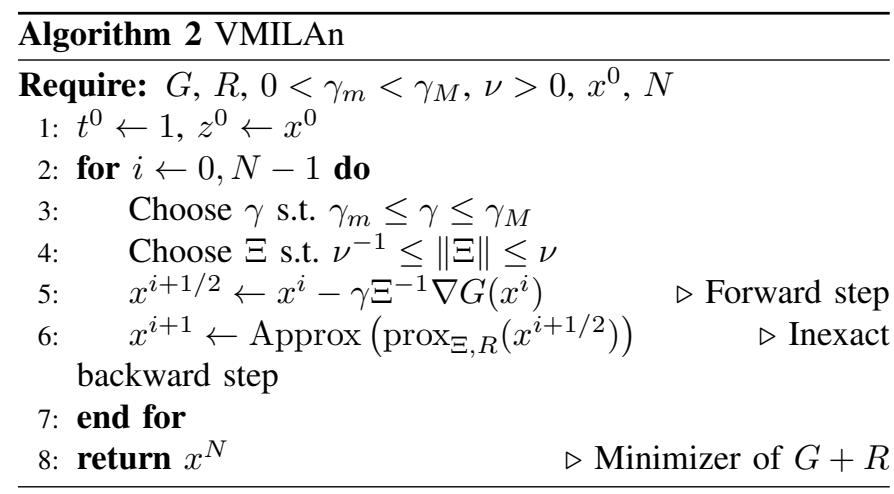

\section{ProMeSCT}

We show how VMILAn may be used for the spectral PCCT problem (3) which defines the ProMeSCT algorithm. In the process we verify the conditions of convergence stated above.

1) Fit to data-term: For every $(p, m)$, the function: $A \in$ $[0,+\infty)^{L \times K} \rightarrow\left(e^{-S A \Sigma} F\right)_{p, m} \in(0,+\infty)$ is real-analytic, so are the square function and the logarithm on its domain and thereby so are each terms of $G_{G}$ and $G_{P}$. Hence $G_{G}$ and $G_{P}$ both have the $\mathrm{K} \notin$ property since they are real-analytic.

\footnotetext{
${ }^{1} G$ is smooth and has the KŁ property, $R$ is non-smooth but convex proper lower semi-continuous, and the steps $\gamma_{i}$ and the spectra of the metric $\Xi_{i}$ are uniformly bounded above and below.
}

We will need the gradients of these functions:

$$
\begin{aligned}
& \nabla G_{G}(A)=2 S^{T}\left(\left[Y-e^{-S A \Sigma} F\right] F^{T} \odot e^{-S A \Sigma}\right) \Sigma^{T} \\
& \nabla G_{P}(A)=-S^{T}\left(\left[1-Y \oslash e^{-S A \Sigma} F\right] F^{T} \odot e^{-S A \Sigma}\right) \Sigma^{T},
\end{aligned}
$$

where $\oslash$ stands for the pointwise division and $\odot$ the pointwise (or Hadamard) product.

2) Regularization: The regularization term $R$ must encode that knowledge we have a priori on the concentration maps $a_{k}$. First one needs to ensure the non-negativity of all the entries as they are concentrations. The convex (but non-differentiable) corresponding indicator function $R_{+}$reads

$$
R_{+}(A)= \begin{cases}0, & \text { if } A_{l, k} \leq 0 \forall l, k, \\ +\infty, & \text { otherwise }\end{cases}
$$

Without metric, the proximal operator of $R_{+}$is simply the projection on non-negative values:

$$
\operatorname{prox}_{R_{+}}(A)_{l, k}= \begin{cases}A_{l, k}, & \text { if } A_{l, k} \geq 0 \\ 0, & \text { otherwise }\end{cases}
$$

Since the proximal operator does not have an analytic expression with a metric, we compute it with a fast proximal iterative scheme FISTA [36] and implement the stopping criterion in VMILAn [29].

One can easily add additional $a$ priori on the spatial concentration maps $a_{k}$ by adding on top of $R_{+}$other convex regularizers and following the exact same procedure. In particular, one can add a TV regularization that will output maps with sharp contours separating smooth parts, or one can also enforce sparsity of the maps (only few points in the maps have nonzero concentrations). This can be done by adding a term on the $l_{1}$-norm of each map as this was done in [38].

3) Choice of the metric: At each iteration of VMILAn, we apply the inverse metric $\Xi_{i}^{-1}$ and compute a metric proximal point $\operatorname{prox}_{\Xi_{i}, R}$. To lighten the computational burden, we choose here to keep the metric constant $\Xi_{i}=\Xi$. Its norm is thus uniformly bounded above and below.

A natural choice for the metric is to include information about the second order derivatives of $G$, while making sure that the metric is positive-definite. The Gauss-Newton approximation of the Hessians of $G_{G}$ and $G_{C}$ are good candidates, but they depend on the current estimate of $A$. However they share a common structure and may both be bounded by the same positive-definite matrix:

$$
\Xi=\Sigma F F^{T} \Sigma^{T} \otimes S^{T} S
$$

(corresponding to the case where $A=0$ ) where $\otimes$ denotes the Kronecker product. This metric separates into two parts. $\Sigma F F^{T} \Sigma^{T}$ preconditions the spectral separation problem and acts on the columns of $A$. It is small $(K \times K)$ and thus easily invertible. $S^{T} S$ is the composition of CT projection and backprojection. It preconditions the reconstruction problem (from sinogram to object space). As noted in [39], it is easily invertible using the conic filter for parallel-beam and fan-beam 
tomography. For the real case of cone-beam tomography, we find that using the conic filter still provides an efficient metric ${ }^{2}$.

4) Gradient step $\gamma$ : We keep the gradient step $\gamma$ constant, which, together with the previous choices, guarantees that the VMILAn converges to a critical point of $J$. Specifically we chose $\gamma$ with respect to the preconditionned vector $\Xi^{-1} \nabla G(A)$ i.e. $\gamma \propto \frac{1}{\| \Xi^{-1}|| \beta}$ with $\beta$ a Lipschitz constant of $\nabla G$. Incorporating the metric designed above (see Eq. (13)) and simplifying leads to $\gamma=\frac{c}{\left\|\Sigma F F^{T} \Sigma^{T}\right\|}$ where $c$ is estimated empirically.

The ProMesCT algorithm is thus the special case of the VMILAn with the specific parameters described above. In the following, the inexact proximal point at step 6 of Algo. 2 is computed using a FISTA scheme and the stopping criterion ensures the convergence of the whole procedure. To demonstrate the efficiency of ProMesCT, we compare it against i) ForwardBackward (whose convergence is also guaranteed but is slow) and ii) FISTA which is applied on $J$ and is not guaranteed to converge in this case.

\section{RESULTS ON SIMULATED DATA}

In this section, we assess ProMeSCT on synthetic data. We compare its performances to FB and FISTA in a first part, then compare its performances to five one-step algorithms of the state of the art in a second part. The performance metric we use is either the value of the concentration of materials, or the Contrast-to-Noise Ratio (CNR) computed from two Regions-Of-Interest (ROI), inside and outside areas with contrast agents, as follows: $\mathrm{CNR}=\left|\overline{\mathbf{a}}_{\text {in }}-\overline{\mathbf{a}}_{\text {out }}\right| / \sqrt{\sigma_{\text {in }}^{2}+\sigma_{\text {out }}^{2}}$ with $\overline{\mathbf{a}}$ the mean of $\mathbf{a}$. We simulate pure Poisson noise and consequently use the discrepancy term $G_{P}$ everywhere.

\section{A. Material}

A cylindrical phantom of size $128 \times 16 \times 128$ with six inserts containing two different contrast agents at different concentrations has been simulated. The contrast agents are iodine (at concentrations 11, 20 and $40 \mathrm{mg} / \mathrm{mL}$, which are biologically relevant for angiography [40], obtained from dilution of IOMERON 400) and silver (at concentrations 11, 20 and $40 \mathrm{mg} / \mathrm{mL}$ obtained from dilution of silver nitrate). Note that the presented setup lies in the context of preclinical imaging. The developed methodology is theoretically compatible with clinical imaging provided that the spectral measurements (with harder beams than in preclinical) captures enough information on the materials of interest, e.g. the contrast agents. The Kedges of silver and iodine are however too low to be considered as contrast agents for clinical imaging. Monochromatic projections of size $186 \times 16$ with $65 \mu \mathrm{m} \times 65 \mu \mathrm{m}$ pixel size have been simulated for a range of energy from 10 to $50 \mathrm{keV}$ with a step of $0.1 \mathrm{keV}$. For each energy, a total number of 720 projections covering a 360 degrees span angle has been computed in a parallel-beam geometry. Finally, five polychromatic scans have been computed according to Eq. (3), which mimic an energy spectrum generated by a tungsten anode X-ray tube

\footnotetext{
${ }^{2}$ In practice, the filter is applied slice by slice in Fourier space. A classical trick to avoid artefacts due to its infinite support is to increase the size of all the data by zero-padding. We neglect this point since the purpose here is to accelerate the process and not to provide an exact invert of the $S^{T} S$ operator.
}

operated at $50 \mathrm{kVp}$ with a filtration with $0.6 \mathrm{~mm}$ of $\mathrm{Al}$. Each acquisition differs from the others according to the minimum energy threshold, i.e. $22,25.5,29,33.2$ and $36.7 \mathrm{keV}$.

In this benchmark, the $\Sigma$ matrix has been filled with the spectral signatures of a solution of iodine at $40 \mathrm{mg} / \mathrm{mL}$, a solution of silver at $40 \mathrm{mg} / \mathrm{mL}$ and the spectral signature of water. The $F$ matrix has been computed with exactly the same spectral responses used for simulating each scan. Fig. 1 displays the lines of the spectral signatures dictionary $\Sigma$ and the columns of the spectral responses of the system matrix $F$. We have assessed FB, FISTA and ProMeSCT (the latter using the metric given in Eq. (13)) on this set of simulated data. We ran 1000 iterations of each algorithm. For the computation of the approximated proximal point in the main ProMeSCT loop (step 6 in Algo. 2), 200 to 1000 iterations of FISTA have been run (to fulfill the stopping condition described in [29]). For all the following simulated and real experiments, the initialization point is $x^{0}=\mathbf{0} \in \mathbb{R}^{L \times K}$ and we use the positivity constraints $R_{+}$as the regularization term.
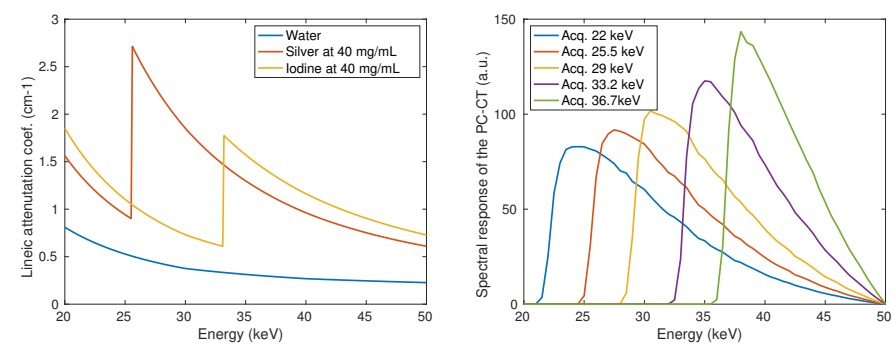

Fig. 1. Left: lines of the $\Sigma$ matrix corresponding to the linear attenuation coefficient of iodine in solution at $40 \mathrm{mg} / \mathrm{mL}$, silver in solution at $40 \mathrm{mg} / \mathrm{mL}$ and water. Right: Normalized columns of the $F$ matrix corresponding to the 5 spectral responses of the 5 simulated tomographic acquisitions.

\section{B. Results on $3 D$ data}

Fig. 2 presents the results obtained in a noisy case with a pure Poisson noise on data with 10000 counts in flat fields for every acquisition. It exhibits first the acceleration that FISTA and ProMeSCT provide with respect to FB. We however recall here that our problem is non convex and does not provide any guarantee of convergence for FISTA. In the case of ProMeSCT, the objective function $J$ decreases monotonically and significantly faster than $\mathrm{FB}$ due to the second order information of the objective function carried out by the metric. The estimation of the concentration of iodine balls along the iterations (Fig. 2) shows that ProMeSCT is more efficient than FISTA and can provide a rough estimation of the concentration very early during the minimization process (this is also true for silver balls although not shown here). In Fig. 2, the error bars show the standard deviation of the pixels values in the ROI. The ROI is a ball inside the iodine ball of radius smaller by three pixels to avoid partial volume effects.

Table I gathers quantitative results at 300 and 1000 iterations. FB remains far from convergence after 1000 iterations, which is quite unsatisfactory. The concentration maps obtained at 1000 iterations reveal that the separation of each of the 3 materials is made almost perfectly and without ambiguity for FISTA and ProMeSCT. No crosstalk can be seen and all the 

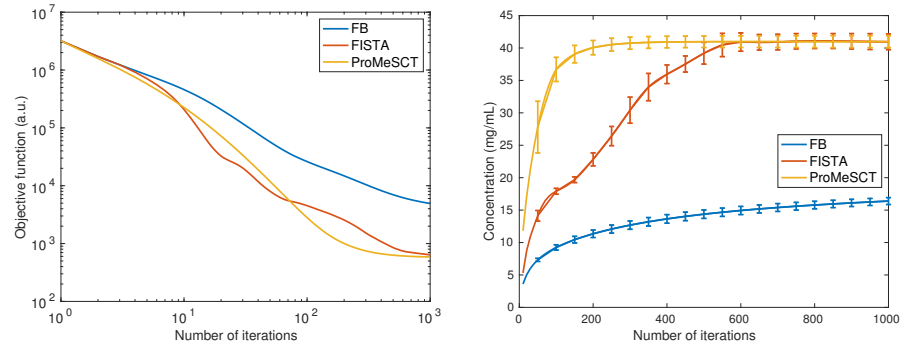

Fig. 2. Objective function $J$ (left) and estimated concentration of the iodine ball at $40 \mathrm{mg} / \mathrm{mL}$ (right) w.r.t. the number of iterations for FB, FISTA and ProMeSCT.

quantities are correctly estimated. Despite the non-convexity of the problem, it seems that in this case both algorithms converge to the same critical point.

An interesting point is to focus on intermediate results displayed on Fig. 3 at 300 iterations. Even if the objective values for FISTA and ProMeSCT are close, the separation of iodine and silver is significantly better with ProMeSCT than with FISTA. Except the most concentrated silver ball, the concentrations of all the other balls are already correctly estimated in the correct material concentration map (iodine or silver) with ProMeSCT. The computation times of each algorithm (in Table I) reveals that despite its apparent higher complexity with respect to FB and FISTA, ProMeSCT is the fastest on this example. It can be explained by the following facts: i) step 6 in Algo. 2 has been implemented on a NVIDIA K20 GPU and ii) an early good quality estimation of sparse iodine and silver concentration maps speeds up computations based on sparse-encoded matrix-vector multiplications.

TABLE I

ESTIMATED CONCENTRATIONS OF CONTRAST AGENTS BY THE THREE PROPOSED METHODS ON SIMULATED DATA AT 300 AND 1000 ITERATIONS AND COMPUTATION TIME.

\begin{tabular}{|c|c|c|c|c|}
\hline & & \multicolumn{3}{|c|}{ silver balls } \\
\hline \multicolumn{2}{|c|}{ Concentration $(\mathrm{mg} / \mathrm{mL})$} & 11 & 20 & 40 \\
\hline FB & 300 iter. & $6.45 \pm 0.20$ & $8.09 \pm 0.32$ & $10.91 \pm 0.57$ \\
\hline FB & 1000 iter. & $7.47 \pm 0.18$ & $9.74 \pm 0.26$ & $14.16 \pm 0.46$ \\
\hline FISTA & 300 iter. & $10.87 \pm 0.51$ & $14.52 \pm 0.65$ & $19.58 \pm 0.81$ \\
\hline FISTA & 1000 iter. & $11.80 \pm 0.71$ & $19.62 \pm 0.85$ & $32.52 \pm 1.38$ \\
\hline ProMeSCT & 300 iter. & $11.76 \pm 1.89$ & $20.54 \pm 2.89$ & $28.78 \pm 7.09$ \\
\hline ProMeSCT & 1000 iter. & $11.57 \pm 2.85$ & $21.71 \pm 2.11$ & $42.43 \pm 0.94$ \\
\hline
\end{tabular}

\begin{tabular}{|c|c|c|c|c|}
\hline & & \multicolumn{3}{|c|}{ iodine balls } \\
\hline \multicolumn{2}{|c|}{ Concentration $(\mathrm{mg} / \mathrm{mL})$} & 11 & 20 & 40 \\
\hline FB & 300 iter. & $7.26 \pm 0.17$ & $9.41 \pm 0.25$ & $12.68 \pm 0.64$ \\
\hline FB & 1000 iter. & $7.87 \pm 0.15$ & $10.93 \pm 0.23$ & $14.15 \pm 0.46$ \\
\hline FISTA & 300 iter. & $10.93 \pm 0.88$ & $17.31 \pm 1.30$ & $30.41 \pm 2.01$ \\
\hline FISTA & 1000 iter. & $11.89 \pm 0.99$ & $24.27 \pm 1.11$ & $32.52 \pm 1.38$ \\
\hline ProMeSCT & 300 iter. & $12.27 \pm 1.21$ & $23.60 \pm 1.37$ & $40.75 \pm 0.93$ \\
\hline ProMeSCT & 1000 iter. & $12.35 \pm 1.23$ & $22.12 \pm 2.03$ & $41.43 \pm 0.94$ \\
\hline
\end{tabular}

\begin{tabular}{|c|ccc|}
\hline & \multicolumn{3}{|c|}{ Computation time in seconds } \\
\hline Algorithm & FB & FISTA & ProMeSCT \\
\hline \hline 300 iterations & 5764 & 5325 & 3437 \\
\hline 1000 iterations & 18442 & 17597 & 11350 \\
\hline
\end{tabular}

\section{Results on $2 D$ data}

In this section, we compare the results of ProMeSCT with five one-step algorithms of the state of the art ([22], [24],
[21], [23], [3]). A comparative study of these algorithms is presented in [26] together with the open-source Matlab toolbox 'OneStepSpectralCT' that can be downloaded online ${ }^{3}$. We refer to this paper for a detailed description of the implementation of each algorithm, and we use a contraction of the first author's name and publication year (e.g. Cai2013) to refer to each method. The 'OneStepSpectralCT' toolbox partly relies on the AIR toolbox ${ }^{4}$ presented in [41] to compute the projection and backprojection operators. Note that the projection operator is computed and stored as a sparse matrix, the backprojection being its transpose. This matrix becomes too large to be computed for 3D volume of size of the one presented in the previous section $(128 \times 16 \times 128)$. This is the reason why we have performed some tests in $2 \mathrm{D}$ for a slice of $128 \times 128$ pixels of the same object that the one presented in the previous section.

Note that all the algorithms implemented in 'OneStepSpectralCT' are using a spatial regularization term designed to enforce the sparsity of the gradients of solutions, whereas our implementation of ProMeSCT encompasses a positivity constraint. For a fair comparison of performances, we did not activate the positivity constraint in ProMeSCT and vanishes the spatial regularization terms in the other algorithms by setting the regularizations parameter to 0 . The code of the Barber2016 method has been modified according the equations 47 to 52 in [24] to remove the Total Variation constraint and minimize the data fidelity. The rest of the parameters are the same as those described in [26]. We have incorporated our algorithm within the 'OneStepSpectralCT' toolbox and ran 5000 iterations of each algorithm except for Barber2016, reported to be slower, for which 20000 iterations have been run. All the methods are initialized with zero-filled material slices. Fig. 5 shows the last iterate computed for each of the methods presented here. Due to the fact that the data fidelity term is not the same for all the algorithms, we will not compare the decreasing of objective functions but rather the normalized $\ell_{2}$ difference relatively to the last iterate computed as in [26]:

$$
\text { Normalized } \ell_{2}\left(x_{n}\right)=\sum_{k=1}^{K} \frac{\sum_{v=1}^{N_{v}}\left(x_{n, v, k}-x_{N_{\mathrm{it}}, v, k}\right)^{2}}{K \sum_{v=1}^{N_{v}} \operatorname{GroundTruth}_{v, k}^{2}}
$$

where $k, v$, and $n$ are respectively the indices for material, voxel and iteration, $N_{v}$ stands for the number of voxels in one reconstructed material map $\left(N_{v}=D / K\right)$ and $N_{\text {it }}$ is the number of iterations. This quantity is displayed in Fig. 4 and illustrates the good behavior and the convergence of the iterates $x_{n}$ over the iterations for each algorithm.

Finally, we display in Fig. 6 the evolution of the concentration of the silver and iodine contrast agents over the course of the iterations with a logarithmic scale on the abscissa. These figures illustrate the good behavior of ProMeSCT, which is among the fastest algorithms together with Mechlem2018 and Long2014 on this test. Note in Fig. 5 the strong improvement of quality of images when taking into account the simple positivity constraint in ProMeSCT that does not require any tuning of parameters.

\footnotetext{
${ }^{3}$ https://github.com/SimonRit/OneStepSpectralCT

${ }^{4}$ https://github.com/jakobsj/AIRToolsII
} 

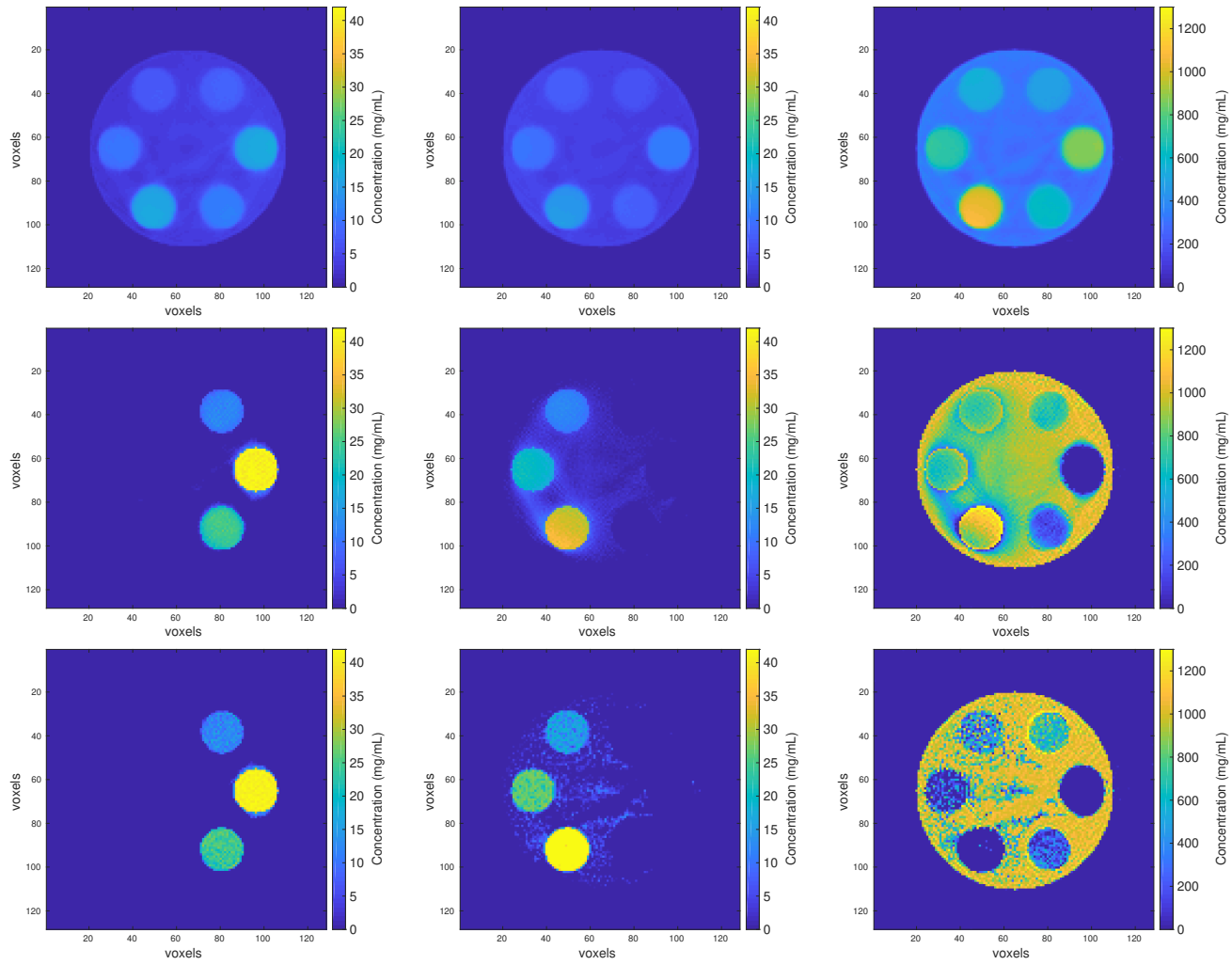

Fig. 3. Concentration maps obtained at 1000 iterations. Top row: FB, middle: FISTA, bottom: ProMeSCT. Left column: iodine, middle: silver, right: water.

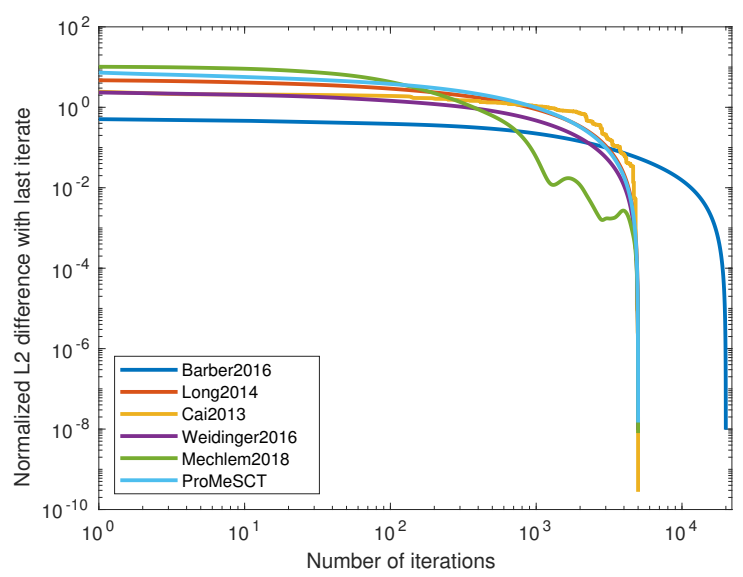

Fig. 4. ROI concentrations of silver (top) and iodine (bottom) over the course of iterations for the five methods referenced in [26] and for ProMeSCT without positivity constraint.

\section{CONCLUSION}

In this paper, we proposed a mathematical framework for the one-step inversion in spectral PC-CT together with an iterative algorithm that is tailored to it. The difficulty of the forward problem lies in its non-linearity, which results in a non-convex inverse problem. The algorithm that is proposed, ProMeSCT, belongs to the family of proximal algorithms. Its specificities are its convergence guarantee for this non-convex problem and its convergence speed, which we were able to exploit by designing a metric that is tailored to the PC-CT. These properties are illustrated on simulated data and compared with state of the art algorithms. On the theoretical side, we plan to investigate further the possible existence of local minima and if they exist, the ability to avoid them by using a particular metric. A nice addition would also be to use ordered subsets to further speed up the process, which raises both theoretical and implementation issues that would be worth pursuing.

\section{ACKNOWLEDGMENTS}

This work was supported by the Région Sud - ProvenceAlpes-Côte d'Azur and partly by the National Infrastructure in Biology and Health "France Life Imaging" (ANR Grant No ANR-11-INBS-0006) of the French Program "Investissements d'Avenir".

\section{REFERENCES}

[1] R. E. Alvarez and A. Macovski, "Energy-selective reconstructions in X-ray computerized tomography," Phys. Med. Biol., vol. 21, no. 5, pp. 733-744, sep 1976.

[2] P. Sukovic and N. H. Clinthorne, "Penalized weighted least-squares image reconstruction for dual energy X-ray transmission tomography," IEEE Trans. Med. Imaging, vol. 19, no. 11, pp. 1075-1081, Nov 2000.

[3] C. Cai, T. Rodet, S. Legoupil, and A. Mohammad-Djafari, "A fullspectral bayesian reconstruction approach based on the material decomposition model applied in dual-energy computed tomography," Med. Phys., vol. 40, no. 11, p. 111916, nov 2013.

[4] W. Wu, F. Liu, Y. Zhang, Q. Wang, and H. Yu, "Non-local low-rank cube-based tensor factorization for spectral CT reconstruction," IEEE Trans. Med. Imaging, vol. 38, no. 4, pp. 1079-1093, apr 2019.

[5] K. Taguchi and J. Iwanczyk, "Vision 20/20: single photon counting X-ray detectors in medical imaging," Med. Phys., vol. 40, no. 10, p. 100901 , oct 2013

[6] F. Cassol et al., "Characterization of the imaging performance of a micro-CT system based on the photon counting XPAD3/Si hybrid pixel detectors," Biomed. Phys. Eng. Express, vol. 2, no. 2, p. 025003, feb 2016. 
[7] _ " "Tracking dynamics of spontaneous tumours in mice using photon counting computed tomography," iScience, vol. 21, pp. 68-83, nov 2019.

[8] L. A. Feldkamp, L. C. Davis, and J. W. Kress, "Practical cone-beam algorithm," J. Opt. Soc. Am. A, vol. 1, no. 6, pp. 612-619, Jun 1984.

[9] H. Erdoğan and J. Fessler, "Monotonic algorithms for transmission tomography," IEEE Trans. Med. Imaging, vol. 18, no. 9, pp. 801-814, 1999.

[10] S. Anthoine, J.-F. Aujol, Y. Boursier, and C. Mélot, "Some proximal methods for Poisson intensity CBCT and PET," Inverse Probl. Imag., vol. 6, no. 4, pp. 565-598, nov 2012.

[11] S. J. Riederer and C. A. Mistretta, "Selective iodine imaging using Kedge energies in computerized X-ray tomography," Med. Phys., vol. 4, no. 6, pp. 474-481, 1977.

[12] F. Cassol et al., "First K-Edge Imaging with a Micro-CT based on the XPAD3 Hybrid Pixel Detector," IEEE Trans. Nucl. Sci., vol. 60, no. 1, pp. 103-108, 2013.

[13] C. T. Badea, S. M. Johnston, Y. Qi, K. Ghaghada, and G. A. Johnson, "Dual-energy micro-CT imaging for differentiation of iodineand gold-based nanoparticles," in Proc. SPIE 7961, Medical Imaging 2011: Physics of Medical Imaging, mar 2011, pp. 555 - 564. [Online]. Available: https://doi.org/10.1117/12.878043

[14] Y. Yuan, Y. Zhang, and H. Yu, "Optimization of energy combination for gold-based contrast agents below $k$-edges in dual-energy micro-ct," IEEE Trans. Rad. Plasma Med. Science, vol. 2, no. 3, pp. 187-193, may 2018.

[15] H. Gao, H. Yu, S. Osher, and G. Wang, "Multi-energy CT based on a prior rank, intensity and sparsity model (PRISM)," Inverse Probl., vol. 27, no. 11, p. 115012, oct 2011.

[16] D. S. Rigie and P. J. L. Rivière, "Joint reconstruction of multi-channel, spectral CT data via constrained total nuclear variation minimization," Phys. Med. Biol., vol. 60, no. 5, pp. 1741-1762, feb 2015.

[17] J. Toivanen, A. Meaney, S. Siltanen, and V. Kolehmainen, "Joint reconstruction in low dose multi-energy ct," Inverse Probl. Imag., vol. 14, no. 4, pp. 607 - 629, aug 2020. [Online]. Available: http://aimsciences.org//article/id/424dbcfa-3088-4585-8f64f2551fa70d0c

[18] J. P. Schlomka et al., "Experimental feasibility of multi-energy photoncounting K-edge imaging in pre-clinical computed tomography," Phys. Med. Biol., vol. 53, no. 15, pp. 4031-404, jul 2008.

[19] M. Dupont et al., "Component Separation for Spectral X-Ray Imaging Using the XPAD3 Hybrid Pixel Camera," in Conf. Rec. IEEE Nucl. Sci. Symp. \& Med. Imag. Conf. 2013, oct 2013, pp. 1-5.

[20] N. Ducros et al., "Regularization of nonlinear decomposition of spectral X-ray projection images." Med. Phys., vol. 44, no. 9, pp. 174-187, sep 2017.

[21] T. Weidinger et al., "Polychromatic iterative statistical material image reconstruction for photon-counting computed tomography," Int. J. Biomed. Imaging, mar 2016.

[22] K. Mechlem et al., "Joint statistical iterative material image reconstruction for spectral computed tomography using a semi-empirical forward model," IEEE Trans. Med. Imaging, vol. 37, no. 1, pp. 68-80, jan 2018.

[23] Y. Long and J. A. Fessler, "Multi-material decomposition using statistical image reconstruction for spectral CT," IEEE Trans. Med. Imaging, vol. 33, no. 8, pp. 1614-1626, aug 2014.

[24] R. F. Barber, E. Y. Sidky, T. G. Schmidt, and X. Pan, "An algorithm for constrained one-step inversion of spectral CT data," Phys. Med. Biol., vol. 61, no. 10, pp. 3784-3818, apr 2016.

[25] B. Chen, Z. Zhang, E. Y. Sidky, D. Xia, and X. Pan, "Image reconstruction and scan configurations enabled by optimization-based algorithms in multispectral CT," Phys. Med. Biol., vol. 62, no. 22, pp. 8763-8793, nov 2017.

[26] C. Mory et al., "Comparison of five one-step reconstruction algorithms for spectral CT," Phys. Med. Biol., vol. 63, no. 23, p. 235001, nov 2018.

[27] S. Tilley, W. Zbijewski, and J. Webster Stayman, "Model-based material decomposition with a penalized nonlinear least-squares CT reconstruction algorithm," Phys. Med. Biol., vol. 64, no. 3, p. 035005, jan 2019.

[28] N. Parikh and S. Boyd, "Proximal algorithms," Found. Trends Optim., vol. 1, no. 3, pp. 127-239, jan 2014.

[29] S. Bonettini et al., "On the convergence of a linesearch based proximalgradient method for nonconvex optimization," Inverse Probl., vol. 33, no. 5 , p. 055005 , mar 2017.

[30] E. Chouzenoux, J.-C. Pesquet, and A. Repetti, "Variable metric forwardbackward algorithm for minimizing the sum of a differentiable function and a convex function," J Optim Theory Appl, vol. 162, no. 1, pp. 107132, 2014.
[31] P. L. Combettes and J.-C. Pesquet, "Proximal splitting methods in signal processing," in Fixed-Point Algorithms for Inverse Problems in Science and Engineering, H. H. Bauschke, R. S. Burachik, P. L. Combettes, V. Elser, D. R. Luke, and H. Wolkowicz, Eds. New York, NY: Springer New York, 2011, ch. 10, pp. 185-212.

[32] K. Kurdyka, "On gradients of functions definable in o-minimal structures," Annales de l'institut Fourier, vol. 48, no. 3, pp. 769-783, 1998.

[33] S. Lojasiewicz, "Une propriété topologique des sous-ensembles analytiques réels," Editions du centre National de la Recherche Scientifique, pp. 87-89, 1963.

[34] P. Combettes and V. Wajs, "Signal recovery by proximal forwardbackward splitting," Multiscale Model. Sim., vol. 4, no. 4, pp. 11681200, 2005.

[35] Y. Nesterov, "Smooth minimization of non-smooth functions," Math. Program., vol. 103, no. 1, pp. 127-152, 2005.

[36] A. Beck and M. Teboulle, "A fast iterative shrinkage-thresholding algorithm with application to wavelet-based image deblurring," in Conf. Rec. IEEE ICASSP, 2009, pp. 693-696.

[37] A. Chambolle and C. H. Dossal, "On the Convergence of the Iterates of the "Fast Iterative Shrinkage/Thresholding Algorithm"," J Optim Theory Appl, vol. 166, no. 3, pp. 968-982, aug 2015.

[38] S. Tairi, "Développement de méthodes itératives pour la reconstruction en tomographie spectrale," Ph.D. dissertation, Aix-Marseille Université, 2019. [Online]. Available: http://www.theses.fr/2019AIXM0160

[39] J. A. Fessler, "Image reconstruction: algorithms and analysis," 2002.

[40] P. He, B. Wei, W. Cong, and G. Wang, "Optimization of k-edge imaging with spectral ct," Med. Phys., vol. 39, no. 11, pp. 6572-6579, 112012.

[41] P. C. Hansen and J. S. Jørgensen, "Air tools ii: algebraic iterative reconstruction methods, improved implementation," Numerical Algorithms, vol. 79, no. 1, pp. 107-137, 2018. [Online]. Available: https://doi.org/10.1007/s11075-017-0430-x 

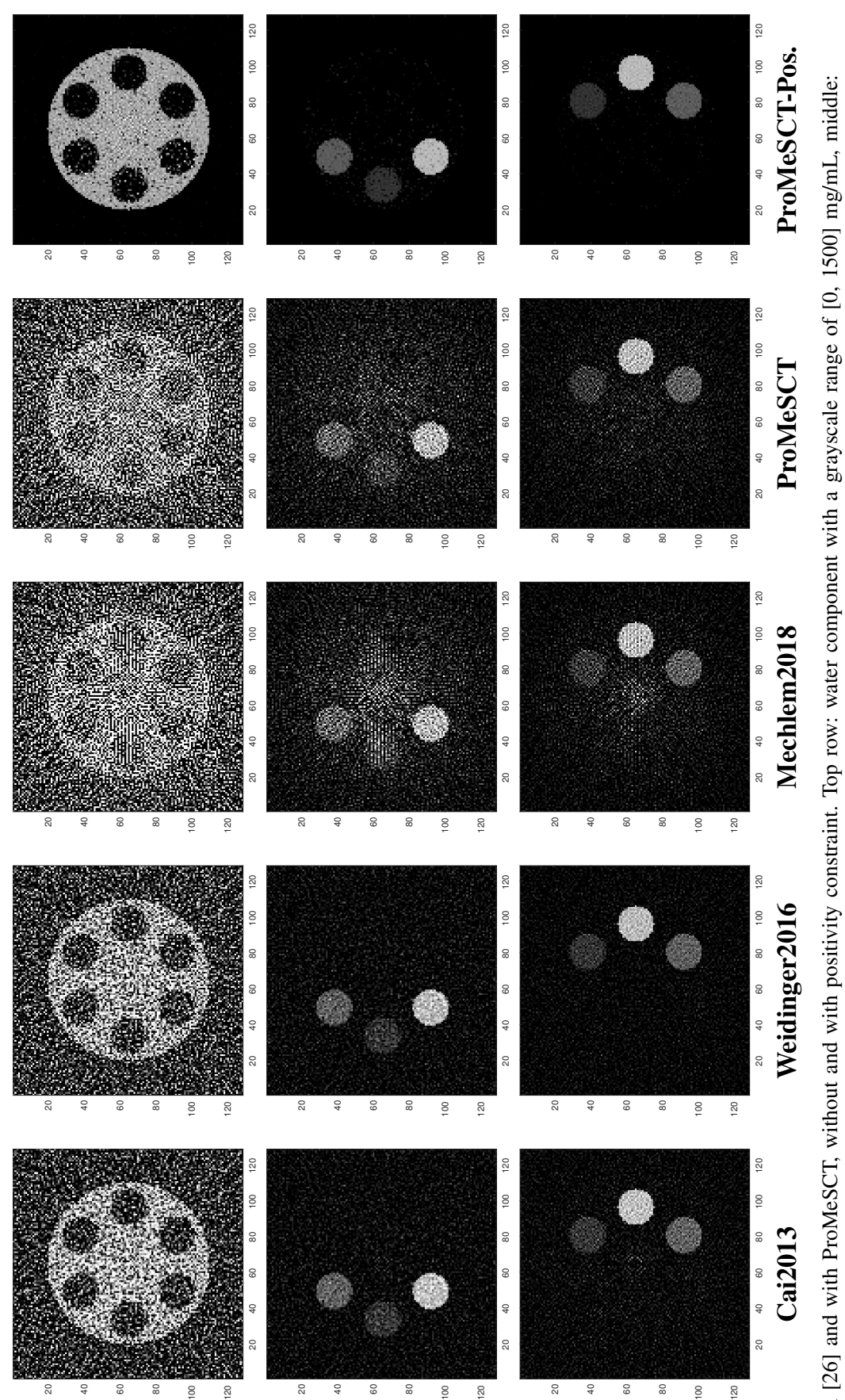

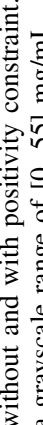
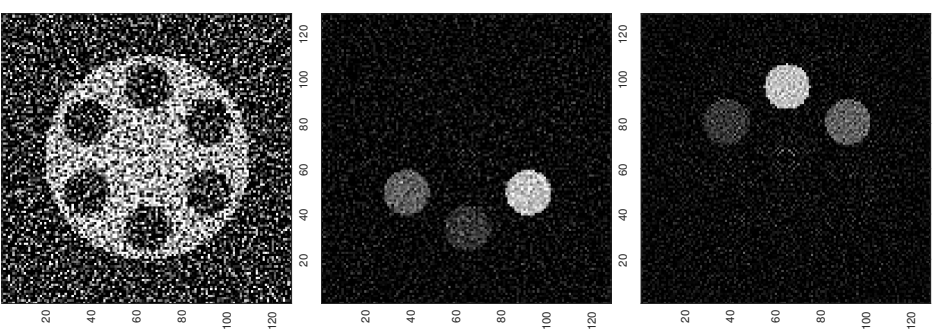

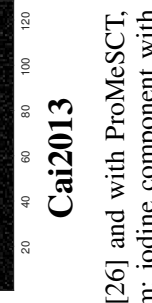
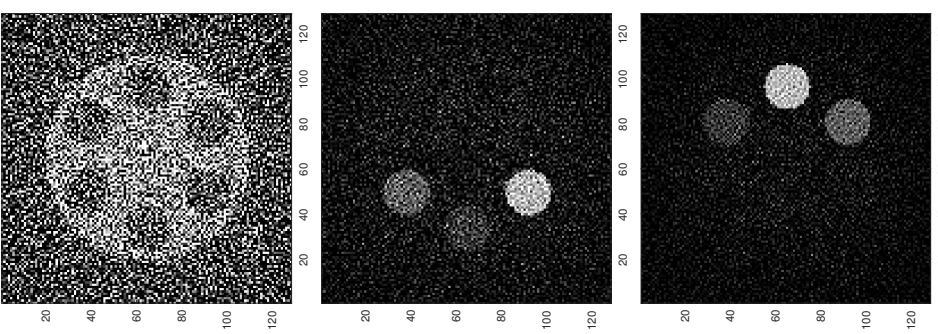

-

형

v



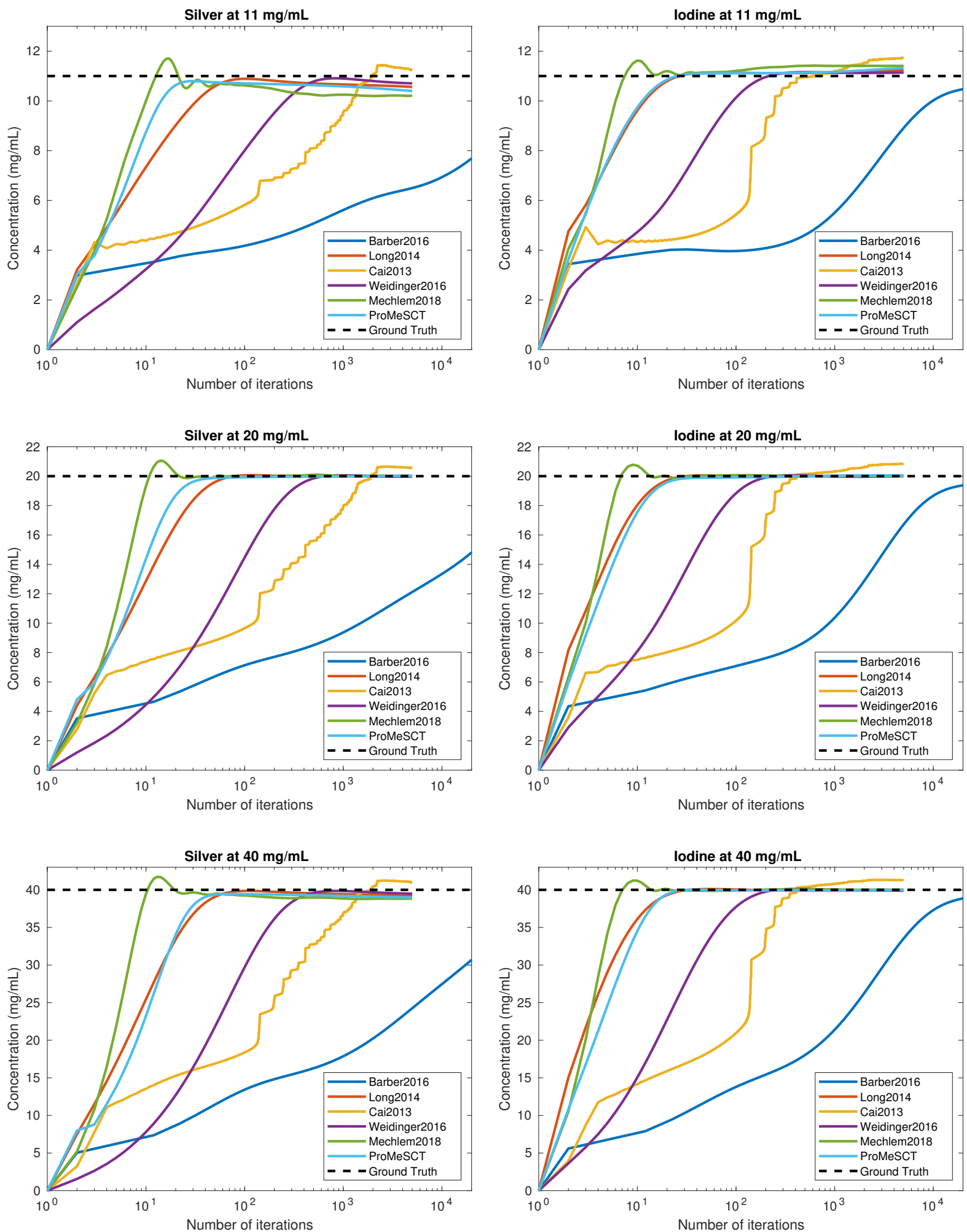

Fig. 6. ROI concentrations of silver (left) and iodine (right) over the course of iterations for the five methods references in [26] and for ProMeSCT without positivity constraint. 